Comment. Math. Helv. 72 (1997) 582-592

(C) 1997 Birkhäuser Verlag, Basel

$0010-2571 / 97 / 040582-11 \$ 1.50+0.20 / 0$

Commentarii Mathematici Helvetici

\title{
The zero-norm subspace of bounded cohomology
}

Teruhiko Soma

\begin{abstract}
Let $\Sigma$ be a closed, orientable surface of genus $>1$. In this paper, non-trivial elements $\alpha$ of the third bounded cohomology $H_{b}^{3}(\Sigma ; \mathbf{R})$ with $\|\alpha\|=0$ are given constructively by using both a hyperbolic metric and a singular euclidean metric on $\Sigma \times \mathbf{R}$. Furthermore, it is shown that the dimension of the subspace $N^{3}(\Sigma)$ of $H_{b}^{3}(\Sigma ; \mathbf{R})$ consisting of zero-norm elements is the cardinality of the continuum.
\end{abstract}

Mathematics Subject Classification (1991). 57M50, 55N99.

Keywords. Bounded cohomology, hyperbolic structures, measured foliations.

\section{Introduction}

Let $X$ be a topological space and $C^{k}(X)$ the $k$-cochain group of real coefficient. The $\mathbf{R}$-subspace $C_{b}^{k}(X)$ of $C^{k}(X)$ consists of elements $c \in C^{k}(X)$ with

$$
\|c\|=\sup \left\{|c(\sigma)| ; \sigma: \Delta^{k} \longrightarrow X \text { is a singular } k \text {-simplex }\right\}<\infty .
$$

Consider the restriction $\delta_{b}^{k}=\left.\delta^{k}\right|_{C_{b}^{k}(X)}: C_{b}^{k}(X) \longrightarrow C_{b}^{k+1}(X)$ of the coboundary operator $\delta^{k}: C^{k}(X) \longrightarrow C^{k+1}(X)$. Then, the cochain complex $\left(C_{b}^{*}(X), \delta_{b}^{*}\right)$ defines the bounded cohomology

$$
H_{b}^{*}(X ; \mathbf{R})=Z_{b}^{*}(X) / B_{b}^{*}(X),
$$

where $Z_{b}^{k}(X)=\operatorname{Ker}\left(\delta_{b}^{k}\right), B_{b}^{k}(X)=\operatorname{Im}\left(\delta_{b}^{k-1}\right)$. We refer to Gromov [7] for fundamental results on bounded cohomology. The pseudonorm $\|\alpha\|$ of $\alpha \in H_{b}^{k}(X ; \mathbf{R})$ is defined by

$$
\|\alpha\|=\inf \left\{\|c\| ; c \in Z_{b}^{k}(X) \text { with }[c]=\alpha\right\} .
$$

We say that $N^{k}(X)=\left\{\alpha \in H_{b}^{k}(X ; \mathbf{R}) ;\|\alpha\|=0\right\}$ is the zero-norm subspace of $H_{b}^{k}(X ; \mathbf{R})$. For any topological space $X$, Matsumoto-Morita [9] and Ivanov [8] proved independently that $N^{k}(X)=\{0\}$ whenever $k \leq 2$. At that moment, any examples of non-trivial $N^{k}(X)$ were not known for $k \geq 3$. 
Here, we are mainly concerned with the case where the space $X$ is a closed, connected, orientable surface $\Sigma$ of genus $>1$. Then, the structure of the second bounded cohomology $H_{b}^{2}(\Sigma ; \mathbf{R})$ was studied by Brooks-Series [2], Mitsumatsu [10], Barge-Ghys [1], Epstein-Fujiwara [4] and that of the third $H_{b}^{3}(\Sigma, \mathbf{R})$ by Yoshida [18], Soma [11], [12] and so on. We refer to Grigorchuk [6] for other useful references on bounded cohomology. Furthermore, the author showed in [13] that $N^{3}(\Sigma)$ is non-trivial by invoking Matsumoto-Morita [9, Theorem 2.3]. However, since the proof of their theorem relies on the Hahn-Banach theorem, we could not construct any non-trivial elements of $N^{3}(\Sigma)$ practically.

In this paper, non-trivial elements of $N^{3}(\Sigma)$ are given constructively by using both a hyperbolic metric and a singular euclidean metric on $\Sigma \times \mathbf{R}$, where the latter metric is defined by using a measured foliation associated to a pseudo-Anosov automorphism of $\Sigma$. A combination of these two metrics presents a continuous family $\left\{\left[c_{r, \varepsilon}\right] ; 0<r \leq 1\right\}$ of elements of $N^{3}(\Sigma \times \mathbf{R})$ which are linearly independent in $H_{b}^{3}(\Sigma \times \mathbf{R} ; \mathbf{R}) \cong \bar{H}_{b}^{3}(\Sigma ; \mathbf{R})$, see Theorems 1 and 2 in $\S 2$ for details. In particular, it is shown that the dimension of the $\mathbf{R}$-vector subspace $N^{3}(\Sigma)$ of $H_{b}^{3}(\Sigma ; \mathbf{R})$ is the cardinality of the continuum.

The key fact in our arguments is that the bounded 3-cocycle $c_{r, \varepsilon}$ given in $\S 2$ is the coboundary of a certain unbounded 2-cochain. For the proof, it is crucial that the 3-dimensional euclidean space $\mathbf{E}^{3}$ is the product metric space $\mathbf{E}^{2} \times \mathbf{E}^{1}$. This is the main reason why we use a euclidean metric as well as a hyperbolic metric.

\section{$\S 1$. Euclidean and hyperbolic structures on manifolds}

Let $\Sigma$ be a closed, connected and oriented surface of genus $>1$. A measured foliation $\mathcal{F}$ on $\Sigma$ is a topological foliation with finitely many prong singular points of degree $\geq 3$ and equipped with the transverse measure. The set of singular points of $\mathcal{F}$ is denoted by $S_{\mathcal{F}}$. An orientation-preserving homeomorphism $f: \Sigma \longrightarrow \Sigma$ is called a pseudo-Anosov automorphism if there exists $\lambda=\lambda(f)>1$ and a pair of mutually transverse, measured foliations $\mathcal{F}^{s}, \mathcal{F}^{u}$ with $S_{\mathcal{F}^{s}}=S_{\mathcal{F}^{u}}(=S(f))$ and $f\left(\mathcal{F}^{s}\right)=\lambda^{-1} \mathcal{F}^{s}, f\left(\mathcal{F}^{u}\right)=\lambda \mathcal{F}^{u}$. We refer to [3], [5] and [16] for the existence and fundamental properties of such automorphisms and for typical pictures of $\mathcal{F}^{s(u)}$ near $p \in S(f)$.

Note that the pair of these measured foliations $\mathcal{F}^{u}, \mathcal{F}^{s}$ determines an incomplete, euclidean structure, a smooth structure on $\Sigma^{\circ}=\Sigma-S(f)$. We will define a smooth structure on $\Sigma$ extending that on $\Sigma^{\circ}$. For any $n \in \mathbf{N}$ with $n \geq 3$, the euclidean 2-space $\mathbf{R}^{2}=\mathbf{C} ;(x, y)=x+\sqrt{-1} y$ is divided into the $n$ sectors $V_{1}, \ldots, V_{n}$ such that

$$
V_{k}=\left\{r \exp (\sqrt{-1} \theta) \in \mathbf{C} ; r \geq 0, \frac{2(k-1) \pi}{n} \leq \theta \leq \frac{2 k \pi}{n}\right\}
$$

for $k=1, \ldots, n$. The upper half plane $H=\{z \in \mathbf{C} ; \operatorname{Im}(z) \geq 0\}$ admits the euclidean structure induced from that on $\mathbf{C}=\mathbf{R}^{2}$. Let $\chi_{k}: V_{k} \longrightarrow H$ be the 
homeomorphism defined by

$$
\chi_{k}(r \exp (\sqrt{-1} \theta))=r \exp \left(\sqrt{-1}\left(\frac{n \theta}{2}-(k-1) \pi\right)\right) .
$$

Note that the Jacobian of $\chi_{k}$ with respect to the standard euclidean coordinates on $V_{k}$ and $H$ is the constant $n / 2$. Let $\mathcal{F}_{H}^{s}, \mathcal{F}_{H}^{u}$ be the measured foliation on $H$ such that the set of leaves in $\mathcal{F}_{H}^{s}$ (resp. $\mathcal{F}_{H}^{u}$ ) consists of straight lines parallel to (resp. straight rays orthogonal to) the $x$-axis $\partial H$ and such that the transverse measures are induced from the euclidean metric on $H$. Then, the pair $\left\{\mathcal{F}_{n}^{s}, \mathcal{F}_{n}^{u}\right\}$ of measured foliations on $\mathbf{R}^{2}$ with the prong singular point $(0,0)$ of degree $n$ is defined by

$$
\mathcal{F}_{n}^{s}=\bigcup_{k=1}^{n} \chi_{k}^{-1}\left(\mathcal{F}_{H}^{s}\right), \quad \mathcal{F}_{n}^{u}=\bigcup_{k=1}^{n} \chi_{k}^{-1}\left(\mathcal{F}_{H}^{u}\right)
$$

For a sufficiently small $\varepsilon>0$, there exist mutually disjoint neighborhoods $U_{p}$ of $p \in S(f)$ in $\Sigma$ and homeomorphisms $\varphi_{p}: U_{p} \longrightarrow D(\varepsilon)=\{z \in \mathcal{C} ;|z|<\varepsilon\}$ such that $\varphi_{p}\left(\left.\mathcal{F}^{s}\right|_{U_{p}}\right)=\left.\mathcal{F}_{n}^{s}\right|_{D(\varepsilon)}, \varphi_{p}\left(\left.\mathcal{F}^{u}\right|_{U_{p}}\right)=\left.\mathcal{F}_{n}^{u}\right|_{D(\varepsilon)}$. For $V_{k}(\varepsilon)=\varphi_{p}^{-1}(D(\varepsilon) \cap$ $\left.V_{k}\right)$, the composition $\left.\chi_{k} \circ \varphi_{p}\right|_{V_{k}(\varepsilon)-\{p\}}: V_{k}(\varepsilon)-\{p\} \longrightarrow H-\{0\}$ is a locally isometric embedding if $V_{k}(\varepsilon)-\{p\}$ has the euclidean metric induced from that on $\Sigma^{\circ}$. Regarding $\left\{\left(U_{p}, \varphi_{p}\right) ; p \in S(f)\right\}$ as a family of coordinate systems for $\Sigma$ in $\cup_{p} U_{p}$, one can define the smooth structure on $\Sigma$ extending that on $\Sigma^{\circ}$. Then, $\Sigma \times I$ admits the product smooth structure, where $I$ is the closed interval $[0,1]$. From now on, we identify $\cup_{p} U_{p} \times I$ with $\cup_{p} D_{p}(\varepsilon) \times I$ via $\varphi_{p} \times$ id ${ }_{I}$ 's, where $D_{p}(\varepsilon)$ are copies of $D(\varepsilon)$. Note that the homeomorphism $f: \Sigma \times\{0\} \longrightarrow \Sigma \times\{1\}$ is not a diffeomorphism with respect to this smooth structure. So, we need another smooth structure on $\Sigma \times I$. For any $t$ with $0 \leq t \leq 1$, consider the elliptic half-disk

$$
E_{t}=\left\{(x, y) \in \mathbf{R}^{2} ; \lambda^{2+2 t} x^{2}+\lambda^{2-2 t} y^{2}=\varepsilon^{2}, y \geq 0\right\}
$$

in $H$. Set $W_{p, t}=\bigcup_{k=1}^{n} \chi_{k}^{-1}\left(E_{t}\right) \subset D_{p}(\varepsilon)$, and

$$
X_{p}=\left\{(q, t) ; t \in I, q \in W_{p, t}\right\} \subset D_{p}(\varepsilon) \times I \subset \Sigma \times I .
$$

For simplicity, we denote the product homeomorphism $\chi_{k} \times \operatorname{id}_{I}: V_{k} \times I \longrightarrow H \times I$ by $\widehat{\chi}_{k}$. The homeomorphism $\psi_{p}: X_{p} \longrightarrow D_{p}(\varepsilon / \lambda) \times I$ is defined by

$$
\psi_{p}(q, t)=\widehat{\chi}_{k}^{-1}\left(\lambda^{t} x, \lambda^{-t} y, t\right)
$$

if $q \in \chi_{k}^{-1}\left(E_{t}\right)$ and $\chi_{k}(q)=(x, y)$. By taking $\left\{\left(X_{p}, \psi_{p}\right) ; p \in S(f)\right\}$ as a coordinte system for $\Sigma \times I$ instead of $\left\{\left(U_{p} \times I, \varphi_{p} \times \mathrm{id}_{I}\right) ; p \in S(f)\right\}$, we have a new smooth structure on $\Sigma \times I$, and denote this smooth manifold by $\Sigma \times I^{\text {new }}$. Then, $f: \Sigma \times$ $\{0\}^{\text {new }} \longrightarrow \Sigma \times\{1\}^{\text {new }}$ is a diffeomorphism. In particular, the mapping torus $M=\Sigma \times I^{\text {new }} /\{(x, 0) \sim(f(x), 1)\}$ admits the induced smooth structure. 
Let $\operatorname{Vol}_{(1)}(B)\left(\right.$ resp. $\left.\operatorname{Vol}_{(2)}(B)\right)$ denote the volume of a compact 3-dimensional submanifold $B$ in $X_{p}^{\circ}=X_{p}-\{p\} \times I$ (resp. in $D_{p}(\varepsilon / \lambda) \times I$ ) with respect to the incomplete euclidean metric on $X_{p}^{\circ} \subset \Sigma^{\circ} \times I$ (resp. the standard euclidean metric on $\left.D_{p}(\varepsilon / \lambda) \times I\right)$. Similarly, the areas of subsurfaces $F$ in $X_{p}^{\circ}$ and $D_{p}(\varepsilon / \lambda) \times I$ are denoted by $\operatorname{Area}_{(1)}(F)$ and $\operatorname{Area}_{(2)}(F)$, respectively.

We denote the degree of $\mathcal{F}^{s}$ (or $\mathcal{F}^{u}$ ) at $p \in S(f)$ by $n(p)$. Then, the following lemma holds.

Lemma 1. (i) For any compact 3-dimensional submanifold $B$ of $X_{p}^{\circ}$,

$$
\operatorname{Vol}_{(1)}(B)=\frac{n(p)}{2} \operatorname{Vol}_{(2)}\left(\psi_{p}(B)\right) .
$$

(ii) For any compact subsurface $F$ of $X_{p}^{\circ}$,

$$
\operatorname{Area}_{(1)}(F) \leq \frac{n(p) \lambda}{2} \operatorname{Area}_{(2)}\left(\psi_{p}(F)\right)
$$

Proof. Since $X_{k}^{\circ} \subset D_{p}(\varepsilon)^{\circ} \times I=\bigcup_{k=1}^{n} V_{k}(\varepsilon)^{\circ} \times I$, if necessary dividing $B$ and $F$ into smaller pieces, we may assume that $B$ and $F$ are contained in $V_{k}(\varepsilon)^{\circ} \times I$ for some $k \in\{1, \ldots, n(p)\}$, where $V_{k}(\varepsilon)^{\circ}=D_{p}(\varepsilon) \cap V_{k}-\{p\}$. Set $B^{\prime}=\widehat{\chi}_{k}(B)$ and $F^{\prime}=\widehat{\chi}_{k}(F)$. Recall that $\left.\widehat{\chi}_{k}\right|_{V_{k}(\varepsilon)^{\circ} \times I}: V_{k}(\varepsilon)^{\circ} \times I \longrightarrow(H-\{0\}) \times I$ is a locally isometric embedding if $V_{k}(\varepsilon)^{\circ}$ has the incomplete euclidean metric induced from that on $\Sigma^{\circ}$. For the diffeomorphism $\Psi: H \times I \longrightarrow H \times I$ with $\Psi(x, y, t)=$ $\left(\lambda^{t} x, \lambda^{-t} y, t\right)$, we have

$$
\begin{aligned}
\operatorname{Vol}_{(1)}(B) & =\operatorname{Vol}_{H \times I}\left(B^{\prime}\right)=\operatorname{Vol}_{H \times I}\left(\Psi\left(B^{\prime}\right)\right) \text { and } \\
\operatorname{Area}_{(1)}(F) & =\operatorname{Area}_{H \times I}\left(F^{\prime}\right) \leq \lambda \operatorname{Area}_{H \times I}\left(\Psi\left(F^{\prime}\right)\right) .
\end{aligned}
$$

Since $\hat{\chi}_{k}\left(\psi_{p}(B)\right)=\Psi\left(B^{\prime}\right)$ and $\hat{\chi}_{k}\left(\psi_{p}(F)\right)=\Psi\left(F^{\prime}\right)$ and since the Jacobian of $\chi_{k}: V_{k} \longrightarrow H$ is $n(p) / 2$, we have

$$
\frac{n(p)}{2} \operatorname{Vol}_{(2)}\left(\psi_{p}(B)\right)=\operatorname{Vol}_{H \times I}\left(\Psi\left(B^{\prime}\right)\right) \text { and } \frac{n(p)}{2} \operatorname{Area}_{(2)}\left(\psi_{p}(F)\right) \geq \operatorname{Area}_{H \times I}\left(\psi\left(F^{\prime}\right)\right) .
$$

This completes the proof.

Let $\rho: \widetilde{M}=\Sigma \times \mathbf{R} \longrightarrow M$ be the infinite cyclic covering associated to $\pi_{1}(\Sigma) \subset$ $\pi_{1}(M)$, and set $\widetilde{L}=S(f) \times \mathbf{R}$. Note that $\widetilde{M}^{\circ}=\Sigma^{\circ} \times \mathbf{R}$ has the product, incomplete euclidean metric induced from the euclidean metrics on $\Sigma^{\circ}$ and $\mathbf{R}$. For the euclidean area form $\eta_{\Sigma^{\circ}}$ on $\Sigma^{\circ}, \widetilde{\eta}=\zeta^{*}\left(\eta_{\Sigma^{\circ}}\right)$ is a 2 -form on $\widetilde{M}^{\circ}$, where $\zeta: \Sigma^{\circ} \times \mathbf{R} \longrightarrow \Sigma^{\circ}$ is the orthogonal projection. The volume form $\widetilde{\Omega}_{E}$ on $\widetilde{M}^{\circ}$ is 
given by $\widetilde{\Omega}_{E}=\widetilde{\eta} \wedge d t$. The diffeomorphism $\widetilde{f}: \widetilde{M} \longrightarrow \widetilde{M}$ with $\widetilde{f}(x, t)=(f(x), t+$ $1)$ is the generator of the covering transformation group. Since $\left.f\right|_{\Sigma^{\circ}}: \Sigma^{\circ} \longrightarrow$ $\Sigma^{\circ}$ is a euclidean-area-preserving diffeomorphism, $\left.\widetilde{f}\right|_{\widetilde{M}^{\circ}}$ is a volume-preserving diffeomorphism, that is, $\widetilde{f}^{*}\left(\widetilde{\Omega}_{E}\right)=\widetilde{f}^{*}(\widetilde{\eta}) \wedge \widetilde{f}^{*}(d t)=\widetilde{\eta} \wedge d t=\widetilde{\Omega}_{E}$. Thus, there exists the 3-form $\Omega_{E}$ in $M^{\circ}=M-L$ with $\rho^{*}\left(\Omega_{E}\right)=\widetilde{\Omega}_{E}$, where $L=\rho(\widetilde{L})$ is a link in $M$. Similarly, there exists a 2 -form $\eta_{M^{\circ}}$ on $M^{\circ}$ with $\rho^{*}\left(\eta_{M^{\circ}}\right)=\widetilde{\eta}$. According to Thurston [17] (see also Sullivan [14]), the smooth manifold $M$ admits a hyperbolic structure. For the hyperbolic volume form $\Omega_{H}$ on $M$, there exists a positive, smooth function $h: M^{\circ} \longrightarrow \mathbf{R}$ with $\Omega_{E}=h \Omega_{H}$. We suppose that $\widetilde{M}$ admits the hyperbolic metric induced from that on $M$ via $\rho$.

For the derivative $d \xi$ of the smooth embedding

$$
\xi=\bigcup_{p \in S(f)} \psi_{p}^{-1}: \bigcup_{p \in S(f)} D_{p}(\varepsilon / \lambda) \times I \longrightarrow \Sigma \times I^{\text {new }} \subset \widetilde{M}
$$

we set

$\iota(\xi)=\inf \left\{\left\|d \xi_{x}(v)\right\|_{\widetilde{M}} ; x \in \bigcup_{p \in S(f)} D_{p}(\varepsilon / \lambda) \times I, v \in T U_{x}\left(\bigcup_{p \in S(f)} D_{p}(\varepsilon / \lambda) \times I\right)\right\}>0$

where $T U\left(\bigcup_{p \in S(f)} D_{p}(\varepsilon / \lambda) \times I\right)$ is the unit tangent bundle over the euclidean manifold $\bigcup_{p \in S(f)} D_{p}(\varepsilon / \lambda) \times I$. We note that the image $Y=\rho\left(\bigcup_{p \in S(f)} D_{p}(\varepsilon / \lambda) \times I\right)$ is a union of solid tori in $M$, and the complement $M-\operatorname{int} Y$ of int $Y$ is a compact manifold.

Lemma 2. $K_{1}=\sup \left\{h(s) ; s \in M^{\circ}\right\}<\infty$.

Proof. For any compact 3-dimensional submanifold $B$ of $Y-L$, we have $\iota(\xi)^{3} \operatorname{Vol}_{(2)}(\widetilde{B}) \leq \operatorname{Vol}_{M}(B)$, where $\widetilde{B}=\rho^{-1}(B) \cap\left(\Sigma \times I^{\text {new }}\right)$. Then, by Lemma 1 (i), we have

$$
\sup \left\{h(s) ; s \in M^{\circ}\right\} \leq \max \left\{\max \{h(s) ; s \in M-\operatorname{int} Y\}, \frac{n(f)}{2 \iota(\xi)^{3}}\right\}<\infty
$$

where $n(f)=\max \{n(p) ; p \in S(f)\}$. This completes the proof.

Note that, in general, for a sequence $\left\{s_{m}\right\}$ in $M^{\circ}$ converging to a point in $L$, the $\operatorname{limit}_{\lim _{m \rightarrow \infty}} h\left(s_{m}\right)$ does not exist. Then, we can not extend $h$ to a continuous map on $M$.

Let $Q$ be a 2-dimensional subspace of $T_{s}\left(M^{\circ}\right)$ for $s \in M^{\circ}$. There exists a small, hyperbolic disk $D$ centered at $x_{0} \in \mathbf{H}^{2}$ and an embedding $i_{Q}: D \longrightarrow M$ with $i_{Q}\left(x_{0}\right)=s, i_{Q^{*}}\left(T_{x_{0}}(D)\right)=Q$ and such that $i_{Q}$ is an isometry onto the image $i_{Q}(D)$ which is totally geodesic with respect to the hyperbolic metric on $M$. Let 
$\varphi_{Q}: D^{\circ} \longrightarrow \mathbf{R}$ be the smooth function with $i_{Q}^{*}\left(\eta_{M^{\circ}}\right)=\varphi_{Q} \cdot \eta_{H}$ on $D^{\circ}$, where $D^{\circ}=D-i_{Q}^{-1}(L)$ and $\eta_{H}$ is the hyperbolic area form on $D$. Then, we have

$$
\int_{D^{\circ}}\left|i_{Q}^{*}\left(\eta_{M^{\circ}}\right)\right| \leq \sup \left\{\left|\varphi_{Q}(x)\right| ; x \in D^{\circ}\right\} \operatorname{Area}_{M}\left(D^{\circ}\right),
$$

where $\operatorname{Area}_{M}\left(D^{\circ}\right)\left(=\operatorname{Area}_{M}(D)\right)$ denotes the hyperbolic area of $D^{\circ}$. Intuitively, $\varphi_{Q}\left(x_{0}\right)$ represents the ratio, in the cross section $Q$, of $\eta_{M}$ 。 to the hyperbolic metric at $s \in M$. It is easily seen that there exists the maximum

$$
g(s)=\max \left\{\left|\varphi_{Q}\left(x_{0}\right)\right| ; Q \text { is a 2-dimensional subspace of } T_{s}\left(M^{\circ}\right)\right\},
$$

and $g: M^{\circ} \longrightarrow \mathbf{R}$ is a continuous, non-negative function. The following lemma is proved by the argument similar to that in Lemma 2.

Lemma 3. $K_{2}=\sup \left\{g(s) ; s \in M^{\circ}\right\}<\infty$.

Proof. As in the proof of Lemma 2, for any compact subsurface $F$ of $Y-L$, the inequality $\iota(\xi)^{2} \operatorname{Area}_{(2)}(\widetilde{F}) \leq \operatorname{Area}_{M}(F)$ holds, where $\widetilde{F}=\rho^{-1}(F) \cap\left(\Sigma \times I^{\text {new }}\right)$. If necessary dividing $\widetilde{F}$ into smaller pieces, we may assume that, for the inclusion $i: \widetilde{F} \longrightarrow \Sigma^{\circ} \times I$, the composition $\zeta \circ i$ is injective. Then, by the definition of $\widetilde{\eta}$,

$$
\int_{\widetilde{F}}\left|i^{*}(\widetilde{\eta})\right|=\operatorname{Area}_{\Sigma^{\circ}}(\zeta(\widetilde{F})) \leq \operatorname{Area}_{(1)}(\widetilde{F}) .
$$

By this inequality together with Lemma 1 (ii),

$$
\int_{F}\left|i_{F}^{*}\left(\eta_{M^{\circ}}\right)\right| \leq \operatorname{Area}_{(1)}(\widetilde{F}) \leq \frac{n(f) \lambda}{2 \iota(\xi)^{2}} \operatorname{Area}_{M}(F)
$$

where $i_{F}: F \longrightarrow Y-L \subset M^{\circ}$ is the inclusion. This shows that

$$
\sup \left\{g(s) ; s \in M^{\circ}\right\} \leq \max \left\{\max \{g(s) ; s \in M-\operatorname{int} Y\}, \frac{n(f) \lambda}{2 \iota(\xi)^{2}}\right\}<\infty .
$$

This completes the proof.

By Lemma 2, for any hyperbolically straight 3-simplex $\sigma: \Delta^{3} \longrightarrow \widetilde{M}$,

$$
\int_{\Delta_{\sigma}^{3 \circ}}\left|\sigma^{*}\left(\widetilde{\Omega}_{E}\right)\right|=\int_{\Delta_{\sigma}^{3 \circ}}\left|(\rho \circ \sigma)^{*}\left(\Omega_{E}\right)\right| \leq K_{1} \int_{\Delta_{\sigma}^{3 \circ}}\left|(\rho \circ \sigma)^{*}\left(\Omega_{H}\right)\right|=K_{1} \operatorname{Vol}\left(\Delta_{\sigma}^{3 \circ}\right),
$$

where $\Delta_{\sigma}^{3}$ denotes the 3-simplex $\Delta^{3}$ with the hyperbolic metric induced from that on $\widetilde{M}$ via $\sigma$ and $\Delta_{\sigma}^{3 \circ}=\Delta_{\sigma}^{3}-\sigma^{-1}(\widetilde{L})$. Since the hyperbolic volume $\operatorname{Vol}\left(\Delta_{\sigma}^{3 \circ}\right)=$ $\operatorname{Vol}\left(\Delta_{\sigma}^{3}\right)$ is less than the volume $\mathbf{v}_{3}$ of a regular ideal simplex in $\mathbf{H}^{3}$,

$$
\int_{\Delta_{\sigma}^{3 \circ}}\left|\sigma^{*}\left(\widetilde{\Omega}_{E}\right)\right|<K_{1} \mathbf{v}_{3}
$$


Similarly, by Lemma 3 together with the equation (1.1), for any straight 2-simplex $\tau: \Delta^{2} \longrightarrow \widetilde{M}$,

$$
\int_{\Delta_{\tau}^{2 \circ}}\left|\tau^{*}(\widetilde{\eta})\right|=\int_{\Delta_{\tau}^{2 \circ}}\left|(\rho \circ \tau)^{*}\left(\eta_{M^{\circ}}\right)\right| \leq K_{2} \operatorname{Area}\left(\Delta_{\tau}^{2 \circ}\right),
$$

where $\Delta_{\tau}^{2}$ denotes the 2-simplex $\Delta^{2}$ with the induced hyperbolic metric and $\Delta_{\tau}^{2 \circ}=$ $\Delta_{\tau}^{2}-\tau^{-1}(\widetilde{L})$. Since $\operatorname{Area}\left(\Delta_{\tau}^{2 \circ}\right)=\operatorname{Area}\left(\Delta_{\tau}^{2}\right)<\pi$,

$$
\int_{\Delta_{\tau}^{2 \circ}}\left|\tau^{*}(\widetilde{\eta})\right|<\pi K_{2}
$$

The inequalities (1.2) and (1.3) will be used in the next section.

\section{$\S 2$. Zero-norm elements of bounded cohomology}

For a topological space $X$, the Gromov norm of a singular $k$-chain $z=\sum_{i=1}^{n} a_{i} \sigma_{i}^{k} \in$ $C_{k}(X)$ with real coefficients $a_{i} \in \mathbf{R}$ is defined by

$$
\|z\|=\sum_{i=1}^{n}\left|a_{i}\right| .
$$

Then, for any bounded $k$-cochain $c \in C_{b}^{k}(X)$, we have $|c(z)| \leq\|c\|\|z\|$.

For any $r \geq 0, \varepsilon>0$, consider the continuous functions $\alpha_{r, \varepsilon}: \mathbf{R} \longrightarrow \mathbf{R}$ and $A_{r, \varepsilon}: \mathbf{R} \longrightarrow \mathbf{R}$ given by

$$
\alpha_{r, \varepsilon}(t)=\min \left\{\varepsilon,|t|^{-r}\right\}, \quad A_{r, \varepsilon}(t)=\int_{0}^{t} \alpha_{r, \varepsilon}(u) d u .
$$

Note that $\lim _{t \rightarrow \infty} \alpha_{r, \varepsilon}(t)=0$ if $r>0$ and $\lim _{t \rightarrow \infty} A_{r, \varepsilon}(t)=\infty$ if $r \leq 1$. The compositions of the projection $\widetilde{M}=\Sigma \times \mathbf{R} \longrightarrow \mathbf{R}$ with $\alpha_{r, \varepsilon}, A_{r, \varepsilon}$ are also denoted by $\alpha_{r, \varepsilon}: \widetilde{M} \longrightarrow \mathbf{R}$ and $A_{r, \varepsilon}: \widetilde{M} \longrightarrow \mathbf{R}$, that is, $\alpha_{r, \varepsilon}(p, t)=\alpha_{r, \varepsilon}(t)$ and $A_{r, \varepsilon}(p, t)=$ $A_{r, \varepsilon}(t)$. For a singular $n$-simplex $\tau: \Delta^{n} \longrightarrow \widetilde{M}$, straight $(\tau): \Delta^{n} \longrightarrow \widetilde{M}$ denotes the straight $n$-simplex obtained by straightening $\tau$, see [15, Chapter 6$]$ for details. Let $c_{r, \varepsilon} \in Z^{3}(\widetilde{M})$ be the 3-cycle defined by

$$
c_{r, \varepsilon}(\sigma)=\int_{\Delta_{\text {straight }(\sigma)}^{3 \circ}} \operatorname{straight}(\sigma)^{*}\left(\alpha_{r, \varepsilon} \widetilde{\Omega}_{E}\right)
$$

for any singular 3-simplex $\sigma: \Delta^{3} \longrightarrow \widetilde{M}$. Intuitively, $c_{r, \varepsilon}(\sigma)$ represents the "euclidean" volume with weight $\alpha_{r, \varepsilon}$ of the "hyperbolically" straightened simplex. Since $\max \left\{\left|\alpha_{r, \varepsilon}(t)\right| ; t \in \mathbf{R}\right\}=\varepsilon$, by (1.2),

$$
\left|c_{r, \varepsilon}(\sigma)\right| \leq \varepsilon \int_{\Delta_{\text {straight }(\sigma)}^{3 \circ}}\left|\operatorname{straight}(\sigma)^{*}\left(\widetilde{\Omega}_{E}\right)\right|<\varepsilon K_{1} \mathbf{v}_{3} .
$$


This shows that $c_{r, \varepsilon} \in Z_{b}^{3}(\widetilde{M})$ and $\left\|c_{r, \varepsilon}\right\| \leq \varepsilon K_{1} \mathbf{v}_{3}$.

In Theorem 1, we will show that the class $\left[c_{r, \varepsilon}\right] \in H_{b}^{3}(\widetilde{M} ; \mathbf{R})$ is independent of $\varepsilon$ if $r>0$. However, Theorem 2 implies that $\left[c_{r, \varepsilon}\right]$ strictly depends on $r$ if $0 \leq r \leq 1$.

Theorem 1. If $0 \leq r \leq 1$, then $\left[c_{r, \varepsilon}\right] \neq 0$ in $H_{b}^{3}(\widetilde{M} ; \mathbf{R})$. If $r>0$, then for any $\varepsilon, \varepsilon^{\prime}>0,\left[c_{r, \varepsilon}\right]=\left[c_{r, \varepsilon^{\prime}}\right]$ in $H_{b}^{3}(\widetilde{M} ; \mathbf{R})$. In particular, if $0<r \leq 1$, then $\left[c_{r, \varepsilon}\right]$ is a non-trivial element of $H_{b}^{3}(\widetilde{M} ; \mathbf{R})$ with $\left\|\left[c_{r, \varepsilon}\right]\right\|=0$.

Proof. We set $\Sigma_{n}=\Sigma \times\{n\} \subset \widetilde{M}$ for $n \in \mathbf{Z}$. For a sufficiently small $\delta>0$, let $\widehat{\Sigma}_{0}$ be an oriented surface piecewise smoothly embedded in $\Sigma \times[-\delta, \delta]$ each piece of which is a totally geodesic triangle with respect to the hyperbolic metric on $\widetilde{M}$ and such that $\widehat{\Sigma}_{0}$ is isotopic to $\Sigma_{0}$ in $\Sigma \times[-\delta, \delta]$. Furthermore, we may take $\widehat{\Sigma}_{0}$ so that it satisfies (2.1).

For any $p \in \Sigma, \widehat{\Sigma}_{0}$ meets the line $\zeta^{-1}(p)$ in a single point.

Let $z_{0} \in Z_{2}(\widetilde{M})$ be a 2-cycle representing this hyperbolic triangulation of $\widehat{\Sigma}_{0}$. We set $\widehat{\Sigma}_{n}=\widetilde{f}^{n}\left(\widehat{\Sigma}_{0}\right)$ and $z_{n}=\widetilde{f}_{*}^{n}\left(z_{0}\right)$. Since $z_{n}-z_{0}$ is homologous to zero in $\widetilde{M}$, there exists a 3 -chain $w_{n} \in C_{3}(\widetilde{M})$ consisting of straight 3-simplices and with $\partial w_{n}=z_{n}-z_{0}$. Note that $\widetilde{\eta}=\zeta^{*}\left(\eta_{\Sigma^{\circ}}\right)$ and $\eta_{\Sigma^{\circ}}>0$. Thus, we have

$$
\int_{\widehat{\Sigma}_{n}^{o}} \widetilde{\eta}=\int_{\Sigma^{\circ}} \eta_{\Sigma^{\circ}}=\int_{\widehat{\Sigma}_{n}^{o}}|\widetilde{\eta}|
$$

where $\widehat{\Sigma}_{n}^{\circ}=\widehat{\Sigma}_{n}-\widehat{\Sigma}_{n} \cap \zeta^{-1}(S(f))$ and the second equality is derived from the property (2.1). We denote the value of these integrals by $K_{3}>0$.

If $\left[c_{r, \varepsilon}\right]=0$ in $H_{b}^{3}(\widetilde{M} ; \mathbf{R})$ for some $0 \leq r \leq 1$, then there would exist a bounded 2-cochain $a \in C_{b}^{2}(\widetilde{M})$ with $\delta_{b}^{2}(a)=c_{r, \varepsilon}$. This implies that, for any $n \in \mathbf{N}$,

$$
\left|c_{r, \varepsilon}\left(w_{n}\right)\right|=\left|a\left(z_{n}-z_{0}\right)\right| \leq\|a\|\left(\left\|z_{n}\right\|+\left\|z_{0}\right\|\right)=2\|a\|\left\|z_{0}\right\| .
$$

Since $A_{r, \varepsilon}$ is an increasing function, $A_{r, \varepsilon}(n-\delta) \leq A_{r, \varepsilon} \leq A_{r, \varepsilon}(n+\delta)$ in $\Sigma_{n} \times[-\delta, \delta]$ and $A_{r, \varepsilon} \leq \varepsilon \delta$ in $\Sigma_{0} \times[-\delta, \delta]$. Consider the 2-form $\theta_{r, \varepsilon}=A_{r, \varepsilon} \widetilde{\eta}$ on $\widetilde{M}^{\circ}$. Since $d \theta_{r, \varepsilon}=\alpha_{r, \varepsilon} d t \wedge \widetilde{\eta}=\alpha_{r, \varepsilon} \widetilde{\Omega}_{E}$ and since straight $\left(w_{n}\right)=w_{n}$, the Stokes Theorem shows that

$$
\begin{aligned}
\left|c_{r, \varepsilon}\left(w_{n}\right)\right| & =\left|\int_{\widehat{\Sigma}_{n}^{\circ}} A_{r, \varepsilon} \widetilde{\eta}-\int_{\widehat{\Sigma}_{0}^{\circ}} A_{r, \varepsilon} \widetilde{\eta}\right| \\
& \geq A_{r, \varepsilon}(n-\delta) \int_{\widehat{\Sigma}_{n}^{\circ}}|\widetilde{\eta}|-\varepsilon \delta \int_{\widehat{\Sigma}_{0}^{\circ}}|\widetilde{\eta}| \\
& =\left(A_{r, \varepsilon}(n-\delta)-\varepsilon \delta\right) K_{3} .
\end{aligned}
$$


The condition $0 \leq r \leq 1$ implies that $\lim _{n \rightarrow \infty} A_{r, \varepsilon}(n-\delta)=\infty$ and hence $\lim _{n \rightarrow \infty}\left|c_{r, \varepsilon}\left(w_{n}\right)\right|=\infty$, a contradiction. It follows that $\left[c_{r, \varepsilon}\right]$ is a non-trivial element of $H_{b}^{3}(\widetilde{M} ; \mathbf{R})$ for any $0 \leq r \leq 1$.

For any $\varepsilon, \varepsilon^{\prime}$ with $\varepsilon>\varepsilon^{\prime}>0$, the 2 -cochain $b \in C^{2}(\widetilde{M})$ is given by

$$
b(\tau)=\int_{\Delta_{\text {straight }(\tau)}^{2 \circ}} \operatorname{straight}(\tau)^{*}\left(\theta_{r, \varepsilon}-\theta_{r, \varepsilon^{\prime}}\right)
$$

for any singular 2-simplex $\tau: \Delta^{2} \longrightarrow \widetilde{M}$. Then, the coboundary of $b$ is $\delta^{2}(b)=$ $c_{r, \varepsilon}-c_{r, \varepsilon^{\prime}}$. If $r>0$, then

$$
K_{4}=\max \left\{\left|A_{r, \varepsilon}(t)-A_{r, \varepsilon^{\prime}}(t)\right| ; t \in \mathbf{R}\right\}=\int_{0}^{\left(\varepsilon^{\prime}\right)^{-1 / r}}\left(\alpha_{r, \varepsilon}(u)-\alpha_{r, \varepsilon^{\prime}}(u)\right) d u<\infty .
$$

By (1.3), we have

$$
\begin{aligned}
|b(\tau)| & =\left|\int_{\Delta_{\text {straight }(\tau)}^{2 \circ}} \operatorname{straight}(\tau)^{*}\left(\left(A_{r, \varepsilon}-A_{r, \varepsilon^{\prime}}\right) \widetilde{\eta}\right)\right| \\
& \leq K_{4} \int_{\Delta_{\text {straight }(\tau)}^{2 \circ}}\left|\operatorname{straight}(\tau)^{*}(\widetilde{\eta})\right| \\
& \leq \pi K_{2} K_{4} .
\end{aligned}
$$

This shows that $b \in C_{b}^{2}(\widetilde{M})$ and hence $c_{r, \varepsilon}-c_{r, \varepsilon^{\prime}} \in B_{b}^{3}(\widetilde{M})$ for $r>0$. By the definition of the pseudonorm, for any $\varepsilon^{\prime}>0,\left\|\left[c_{r, \varepsilon}\right]\right\|=\left\|\left[c_{r, \varepsilon^{\prime}}\right]\right\| \leq \varepsilon^{\prime} K_{1} \mathbf{v}_{3}$. Thus, we have $\left\|\left[c_{r, \varepsilon}\right]\right\|=0$ whenever $r>0$.

For two sequences $\left\{a_{n}\right\},\left\{b_{n}\right\}$ with $a_{n}, b_{n}>0(n \in \mathbf{N}), a_{n} \sim b_{n}$ means that

$$
0<\liminf _{n \rightarrow \infty} \frac{a_{n}}{b_{n}} \leq \limsup _{n \rightarrow \infty} \frac{a_{n}}{b_{n}}<\infty .
$$

The notation in the proof of Theorem 1 still works to prove Theorem 2.

Theorem 2. For a fixed $\varepsilon>0$, the elements $\left[c_{r, \varepsilon}\right](0 \leq r \leq 1)$ are linearly independent in $H_{b}^{3}(\widetilde{M} ; \mathbf{R})$.

Proof. We suppose that

$$
\gamma_{1}\left[c_{r_{1}, \varepsilon}\right]+\gamma_{2}\left[c_{r_{2}, \varepsilon}\right]+\cdots+\gamma_{m}\left[c_{r_{m}, \varepsilon}\right]=0
$$

for $0 \leq r_{1}<r_{2}<\cdots<r_{m} \leq 1$. Then, there exists a bounded 2-cochain $a \in C_{b}^{2}(\widetilde{M})$ with

$$
\gamma_{1} c_{r_{1}, \varepsilon}+\gamma_{2} c_{r_{2}, \varepsilon}+\cdots+\gamma_{m} c_{r_{1}, \varepsilon}=\delta_{b}^{2}(a) .
$$


For the straight 3 -chain $w_{n} \in C_{3}(\widetilde{M})$ given as above, we have

$$
\left|\gamma_{1} c_{r_{1}, \varepsilon}\left(w_{n}\right)\right| \leq \sum_{j=2}^{m}\left|\gamma_{j} c_{r_{j}, \varepsilon}\left(w_{n}\right)\right|+\left|\delta_{b}^{2}(a)\left(w_{n}\right)\right| .
$$

The argument similar to that in the proof of Theorem 1 shows that

$$
\left|\gamma_{1}\right| K_{3}\left(A_{r_{1}, \varepsilon}(n-\delta)-\varepsilon \delta\right) \leq \sum_{j=2}^{m}\left|\gamma_{j}\right| K_{3}\left(A_{r_{j}, \varepsilon}(n+\delta)+\varepsilon \delta\right)+2\|a\|\left\|z_{0}\right\|,
$$

and hence

$$
\left|\gamma_{1}\right| \leq \frac{\sum_{j=2}^{m}\left|\gamma_{j}\right|\left(A_{r_{j}, \varepsilon}(n+\delta)+\varepsilon \delta\right)+2 K_{3}^{-1}\|a\|\left\|z_{0}\right\|}{A_{r_{1}, \varepsilon}(n-\delta)-\varepsilon \delta} .
$$

Since $A_{r_{1}, \varepsilon}(n-\delta) \sim n^{1-r_{1}}, A_{r_{j}, \varepsilon}(n+\delta) \sim n^{1-r_{j}}$ if $r_{j}<1$, and $A_{r_{m}, \varepsilon}(n+\delta) \sim \log n$ if $r_{m}=1$, the right hand side of (2.2) converges to zero as $n \rightarrow \infty$. This shows that $\gamma_{1}=0$. Similarly, we have $\gamma_{2}=\cdots=\gamma_{m}=0$. Thus, $\left[c_{r, \varepsilon}\right](0 \leq r \leq 1)$ are linearly independent.

By Theorems 1 and 2, the continuous family $\left\{\left[c_{r, \varepsilon}\right] ; 0<r \leq 1\right\}$ consists of linearly independent elements in $N^{3}(\widetilde{M})$. Since the inclusion $i: \Sigma=\Sigma_{0} \longrightarrow \widetilde{M}$ is a homotopy equivalence, the induced homomorphism $i^{*}:\left(H_{b}^{3}(\widetilde{M} ; \mathbf{R}),\|\cdot\|\right) \longrightarrow$ $\left(H_{b}^{3}(\Sigma ; \mathbf{R}),\|\cdot\|\right)$ is isometrically isomorphic. Thus, we have the following corollary.

Corollary. For any closed, connected, orientable surface $\Sigma$ of genus $>1$, the dimension of the zero-norm subspace $N^{3}(\Sigma)$ of $H_{b}^{3}(\Sigma ; \mathbf{R})$ is the cardinality of the continuum.

\section{References}

[1] J. Barge et E. Ghys, Surfaces et cohomologie bornée, Invent. Math. 92 (1988), 509-526.

[2] R. Brooks and C. Series, Bounded cohomology for surface groups, Topology 23 (1984), $29-36$.

[3] A. Casson and S. Bleiler, Automorphisms of surfaces after Nielsen and Thurston, London Math. Soc. Student Texts 9, Cambridge Univ. Press, Cambridge 1988.

[4] D. Epstein and K. Fujiwara, The second bounded cohomology of word-hyperbolic groups, Topology 36 (1997), 1275-1289.

[5] A. Fathi, F. Laudenbach and V. Poénaru, Travaux de Thurston sur les surfaces, Astérisque no. 66-67, Soc. Math. France, 1979.

[6] R. Grigorchuk, Some results on bounded cohomology, A. Duncan, N. Gilbert, J. Howie (Eds.), Combinatorial and geometric group theory, London Math. Soc. Lecture Notes Series 204, Cambridge Univ. Press, Cambridge 1995, pp. 111-163. 
[7] M. Gromov, Volume and bounded cohomology, Publ. Math. Inst. Hautes Etud. Sci. 56 (1982), 5-100.

[8] N. Ivanov, Second bounded cohomology group, J. Soviet Math. 52 (1990), 2822-2824.

[9] S. Matsumoto and S. Morita, Bounded cohomology of certain groups of homeomorphisms, Proc. Amer. Math. Soc. 94 (1985), 539-544.

[10] Y. Mitsumatsu, Bounded cohomology and $l^{1}$-homology of surfaces, Topology 23 (1984), $465-471$.

[11] T. Soma, Bounded cohomology of closed surfaces, Topology 36 (1997), 1221-1246.

[12] T. Soma, Bounded cohomology and topologically tame Kleinian groups, Duke Math. J. 88 (1997), 357-370.

[13] T. Soma, Existence of non-Banach bounded cohomology, Topology (in press).

[14] D. Sullivan, Travaux de Thurston sur les groupes quasi-fuchsiens et les variétés hyperboliques de dimension 3 fibrés sur $S^{1}$, Séminaire Bourbaki 554, Lecture Notes in Math., vol. 842, Springer Verlag, Berlin and New York 1981, pp. 196-214.

[15] W. Thurston, The geometry and topology of 3-manifolds, Lecture Notes, Princeton Univ., Princeton 1978.

[16] W. Thurston, On the geometry and dynamics of diffeomorphisms of surfaces, Bull. Amer. Math. Soc. 19 (1988), 417-431.

[17] W. Thurston, Hyperbolic structures on 3-manifolds II: Surface groups and 3-manifolds which fiber over the circle, preprint.

[18] T. Yoshida, On 3-dimensional bounded cohomology of surfaces, Homotopy Theory and Related Topics, Advanced Studies in Pure Math., vol. 9, Kinokuniya, Tokyo, 1986, pp. 173-176.

Teruhiko Soma

Department of Mathematical Sciences

College of Science and Engineering

Tokyo Denki University

Hatoyama-machi, Saitama-ken 350-03

Japan

e-mail: soma@r.dendai.ac.jp

(Received: November 26, 1996) 